\title{
Studies on Properties of Polyaniline-Dodecylbenzene Sulfonic Acid Composite Films Synthesized Using Different Oxidants
}

\author{
C. Basavaraja, R. Pierson, and Do Sung Huh* \\ Department of Chemistry and Institute of Functional Materials, Inje University, Gimhae, Gyungnam 621-749, Korea
}

A. Venkataraman and S. Basavaraja

Department of Materials Science, Gulbarga University, Gulbarga, Karnataka, India

Received November 21, 2008; Revised December 18, 2008; Accepted December 19, 2008

\begin{abstract}
Two types of nano composite were obtained by in situ chemical method in polyaniline (PANI)/dodecylbenzenesulfonic acid (DBSA) system depending on the use of either ammonium persulfate (APS) or ferric chloride $\left(\mathrm{FeCl}_{3}\right)$ as the oxidant. In order to study the difference of the two composites in the surface characteristics, thermal stability, and electric properties, the composite films were studied by transmission electron microscopy (TEM), Xray diffraction (XRD), thermogravimetric analysis (TGA), and temperature dependent DC electrical conductivity. The results revealed a large difference in the surface morphology, thermal stability, and the microstructure properties between the two composites, and these differences were considered responsible for the molecular order and conductivity.
\end{abstract}

Keywords: morphology, polyaniline-dodecylbenzene sulfonic acid, self-assembly.

\section{Introduction}

In recent years, the synthesis of aniline-based copolymers has received great attentions. This is because it is possible to prepare copolymers with electrical properties and stability better than polyaniline (PANI) through the simultaneous polymerization of aniline and other aniline derivatives. ${ }^{1-4}$ Nowadays micro and nano structured conducting polymers are preferred because of their unique properties and the potential utility for nanomaterials in nanodevices. ${ }^{5}$ Among the conducting polymers, PANI is the most useful in potential because of its low cost, ease of preparation, excellent environmental stability, and controllable physical and electrochemical properties such as oxidation and protonation. Advances in the development of an inexpensive signal processing system using micro and nanostructures have made the production of a variety of novel biomedical sensors possible. ${ }^{6-11}$ There has been a growing interest in research on the conducting polymer with nanostructures such as nanorods, nanotubes, nanowires, and nanofibers. Many of these applications require bulk quantity materials, enabling an immediate evaluation of the influence of nanostructures on the properties and applications of conducting polymers. Therefore the development of bulk synthesis for conducting polymers is especially important for practical reasons.

*Corresponding Author. E-mail: chemhds@inje.ac.kr
Nanotubes or nanofibers of PANI have been synthesized by using a porous membrane template. ${ }^{12}$ However, control over the morphology of conducting polymers using a simple and effective method still remains scientifically challenging. The polymer surfactant system is a very attractive system. It is widely studied both in solution and in solid state because of its interesting physical properties, such as easier solubility, mesomorphic behavior, nanostructured morphology, and reversible fusibility. ${ }^{13-16}$ DBSA, a well-known surfactant, forms a colloidal particle and acts as a steric stabilizer because of its small size, thus it disperses finely in an aqueous medium. The anilinium-DBSA complex can be achieved by mechanical or solution mixing on the emeraldine base of PANI with DBSA. The DBSA in solution state adsorbs most of the aniline monomers onto their complex network. ${ }^{17,18}$ The long aliphatic chains of DBSA facilitate the process of mixing for the PANI-DBSA complex in a conventional way, and the long hydrocarbon tail of DBSA easily modifies the polymer-polymer interface in the complex.

In this report, PANI-DBSA composites have been synthesized by oxidative chemical polymerization of aniline at low temperature using the initiators of ammonium persulfate (APS) and $\mathrm{FeCl}_{3}$ (FC) to obtain two types of composites. The interfacial interactions and the thermal stability of the two composite films were compared by XRD, TEM and TGA analysis. An effort was made to understand the signif- 
icant variations in the surface characteristics, thermal and electrical behavior of the two composites. From these results we can suggest that the other way of controlling the characteristic properties of PANI-DBSA composites.

\section{Experimental}

AR grade aniline, APS, and FC were purchased from Sigma-Aldrich while DBSA and $N$-methyl-2-pyrrolidinone (NMP) were obtained from Junsei Chemical Co. All solutions were prepared in aqueous media using deionized water. The concentrations of both DBSA and aniline are set at $0.05 \mathrm{M}$, while that of the oxidants APS and FC, $0.075 \mathrm{M}$; these values remained fixed throughout the experiment. The PANI synthesized in the presence of DBSA using the initiator of APS is called PANI-DP and that with FC is PANI-DF. DBSA was initially dissolved in $200 \mathrm{~mL}$ of water to form an aqueous solution. The aniline was then added slowly, after which the medium was stirred continuously for $2 \mathrm{~h}$ to obtain a uniform dispersion of aniline-DBSA complex. ${ }^{18}$ The oxidant was then slowly added, processing the polymerization of aniline at $0-5^{\circ} \mathrm{C}$ for about $8 \mathrm{~h}$ to produce PANI-DP composites. In a similar fashion, the oxidant $\mathrm{FC}$ was added dropwise to the
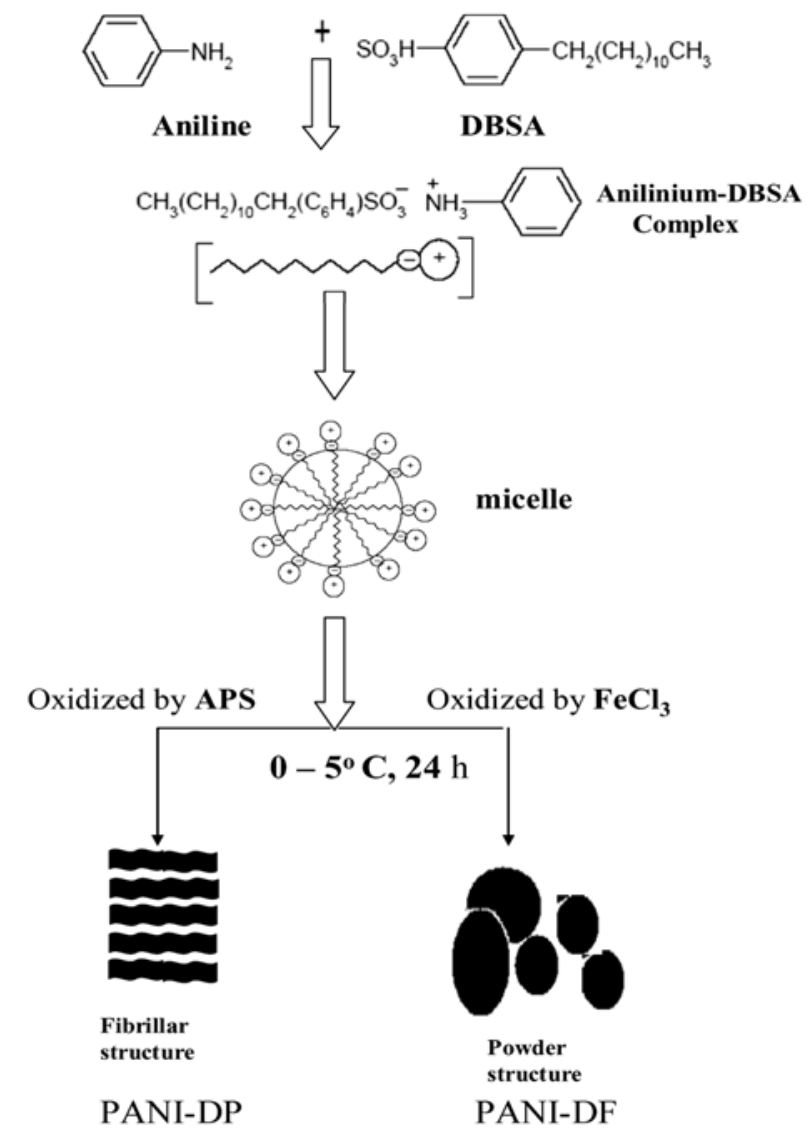

Figure 1. A schematic illustration for the formation of the PANIDP fibrillar structure and the PANI-DF powder form using APS and $\mathrm{FeCl}_{3}$, respectively.
aniline-DBSA dispersion to obtain PANI-DF composites. Precipitated polymer powder was recovered by vacuum filtering and was washed thoroughly with deionized water and acetone. Finally, the resulting powder was dried in a vacuum oven for $24 \mathrm{~h}$ to achieve a constant weight.

Figure 1 shows a schematic illustration for the formation of the composites PANI-DP and PANI-DF by using APS and $\mathrm{FC}$, respectively. The addition of DBSA brings about the formation of anilinium-DBSA complex which comes in the form of a micelle. After adding an oxidant, the aniliniumDBSA complex formed in the aqueous media undergoes different rates of polymerization depending on the kind of oxidant added. In a micelle-aided polymer synthesis, the reaction takes place mainly at the micelle-water interface, and during this process the micelles are in dynamic equilibrium with the surfactant monomers in the solution. ${ }^{17,18}$ During the synthesis the oxidation/reduction potential of PANI might affect the morphology and the size of the micelle in which DBSA is regarded as soft-templates. Under such conditions the micelles present in the solvent not only react but accelerate the polymerization of aniline so that the yield of PANI will have a relatively high molecular weight. The presence of the micelle results in the variation of the morphology and size of the nanostructures. The characterization of the powder samples and the preparation of thin films of the obtained composites were described in our earlier studies. ${ }^{19-21}$ The DC electric measurement of the obtained composite films was performed within the temperature range of 300 - $530 \mathrm{~K}$ using the four-probe technique with a Keithly 224 constant current source and a Keithly 617 digital electrometer.

\section{Results and Discussion}

FTIR Measurements of the Composites. Figure 2 illustrates the FTIR spectra of PANI-DP and PANI-DF. The

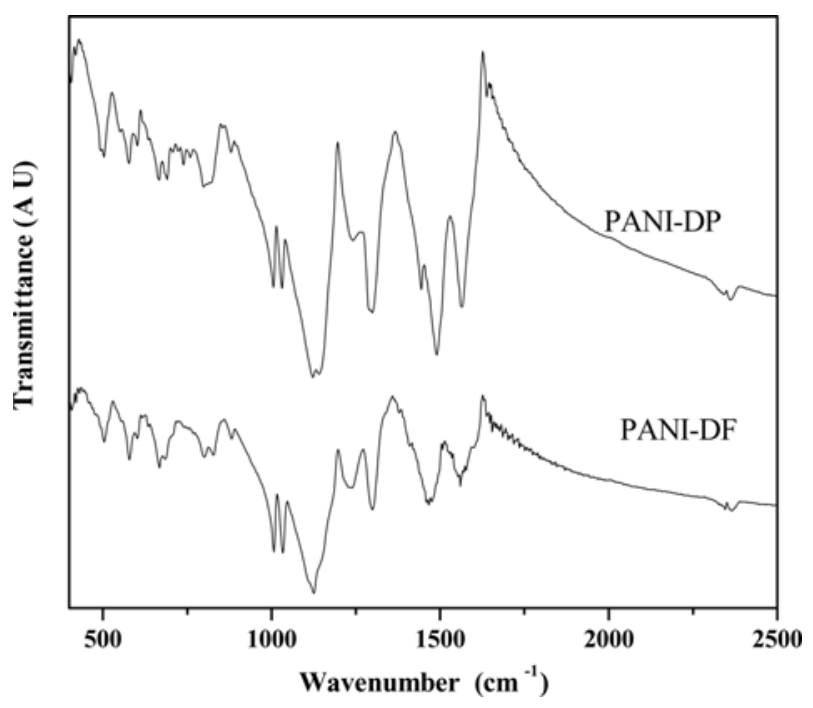

Figure 2. FTIR spectral characterization of PANI-DP and PANI-DF. 
characteristic absorptions of interest are $\mathrm{N}=\mathrm{Q}=\mathrm{N}\left(1165 \mathrm{~cm}^{-1}\right)$, $\mathrm{Q}=\mathrm{N}^{+} \mathrm{H}-\mathrm{B}$ or $\mathrm{B}-{ }^{+} \mathrm{NH}-\mathrm{B}\left(1135 \mathrm{~cm}^{-1}\right),-\mathrm{C}=\mathrm{O}\left(1750 \mathrm{~cm}^{-1}\right)$, and $\mathrm{R}_{-} \mathrm{SO}_{3}^{-}\left(1043 \mathrm{~cm}^{-1}\right)$ for DBSA doping polyaniline, respectively. In particular, the band at $505 \mathrm{~cm}^{-1}$ ascribed to the absorption of $-\mathrm{SO}_{3} \mathrm{H}$ group was observed in both spectra. The disappearance of the main chain $\mathrm{N}=\mathrm{Q}=\mathrm{N}$ absorption and the appearance of characteristic absorptions at around $1130 \mathrm{~cm}^{-1}$ in PANI-DP and $1040 \mathrm{~cm}^{-1}$ in PANI-DF confirm that doping has taken place in both the composites. ${ }^{22} \mathrm{~A}$ slightly shifted peak in PANI-DP and a sharp peak in PANI$\mathrm{DF}$ at $1556 \mathrm{~cm}^{-1}$ indicate Quinoid ring stretching. The C-N stretching of secondary aromatic amine at $1300 \mathrm{~cm}^{-1}$ is present in both the composites which appear as a slightly broadened peak in PANI-DP and as an intensely sharp peak in PANI-DF. A para substituted peak at $798 \mathrm{~cm}^{-1}$ appears as a broad peak in PANI-DF and as a shoulder peak in PANIDP. In PANI-DP the out-of-plane deformation of $\mathrm{C}-\mathrm{H}$ in the 1,4-disubstituted benzene ring $\left(821 \mathrm{~cm}^{-1}\right)$ observed in PANI$\mathrm{DF}$ is absent. Further $\mathrm{C}=\mathrm{C}$ stretching deformation of quinoid at $1594 \mathrm{~cm}^{-1}$ and the peak for the benzenoid ring at $1523 \mathrm{~cm}^{-1}$ are present in PANI-DF but not in PANI-DP.

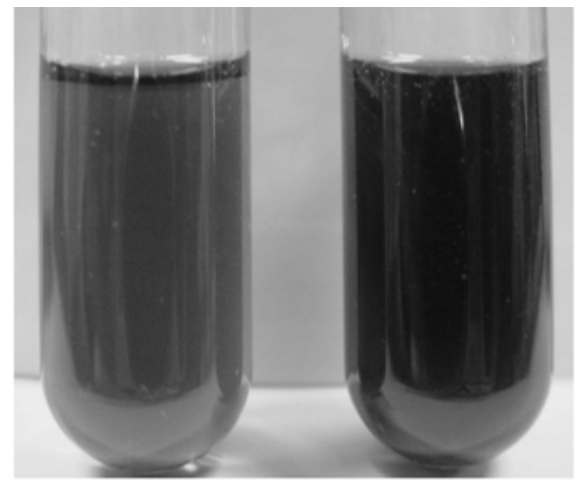

(a)

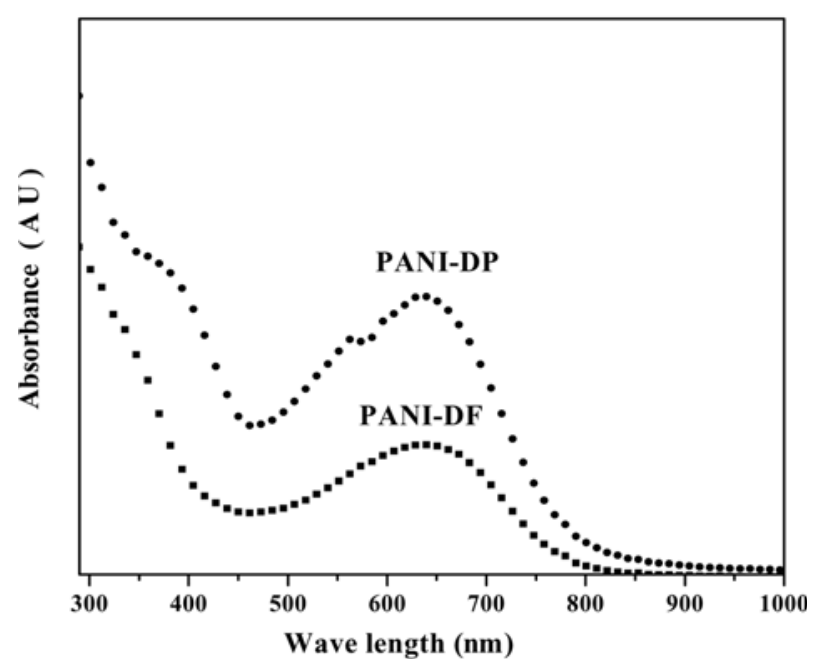

(b)

Figure 3. (a) NMP solutions and (b) UV-Vis spectra of PANI-DP and PANI-DF composites.
UV-Vis Spectral Studies for the Composites. Figure 3(a) demonstrates the color of the composite solutions of PANIDP and PANI-DF in NMP during the study of UV-Vis spectra, which shows a dark green color for PANI-DP and pinkish for PANI-DF. Figure 3(b) shows the UV-Vis spectra of PANI-DP and PANI-DF which were synthesized by using APS and $\mathrm{FeCl}_{3}$ respectively. The spectrum of PANI-DP shows two absorption maxima, one at around 350 and $640 \mathrm{~nm}$ corresponding to the $\pi$ - $\pi^{*}$ and $n-\pi^{*}$ transition of the benzene ring. The disappearance of the peak at $350 \mathrm{~nm}$ in PANI-DF may be attributed to the absence of $\pi-\pi^{*}$ transition of the benzene ring. The broad absorption peak at $640 \mathrm{~nm}$ was a result of $n-\pi^{*}$ transition from the nonbonding nitrogen lone pair to the conduction band. In both the composites the peaks are red-shifted by a factor of 30 and $10 \mathrm{~nm}$ respectively, resulting in the increase in conjugation caused by steric repulsion between PANI and DBSA. ${ }^{23}$ The absence of the extended tail at a higher wavelength $(\approx 800 \mathrm{~nm})$ in both PANI-DP and PANI-DF indicates the coiling of the polymer chain. The peak which is at around $640 \mathrm{~nm}$ in both PANIDP and PANI-DF indicates that the PANI remained in the emeraldine base form in the NMP solution. Because NMP is a highly polar solvent $(\varepsilon \approx 32)$, the solute-solvent interaction is strong ${ }^{24}$ resulting in the conversion of the salt phase into an insulating emeraldine base form. ${ }^{25,26}$ Thus the dark green color observed in PANI-DP and the pale green color seen in PANI-DF in NMP are second proof for the higher doping of DBSA on the backbone of PANI. This will be further evidenced by the thermal studies of these composites to be discussed in subsequent sections.

Micro-Structure Properties. Figures 4(a) and (b) shows the TEM images of PANI-DP and PANI-DF, taken at 100 $\mathrm{nm}$ respectively. The PANI-DP in 4(a) forms a lump like structure, whereas PANI-DF in 4(b) shows an amorphous structure. PANI-DP forms aggregated clusters which are semicrystalline in nature. This suggests that the change in the formation of polymer matrix during the polymerization process. This may be due to the surfactant tails that is crystallized or remain typically disordered in solid state. ${ }^{27}$ The physical and mechanical properties of the complex depend on the structure of the surfactant in the complex as well as on the overall morphology of the blend. From the figures showing the TEM micrograph of the two structures, PANIDP is different from PANI-DF in terms of surface morphology. The changes in the morphology of the composites may be due either to the process in the formation of the composite material in the presence of different oxidizing agents (i.e., APS and FC), or some other kind of rearrangement in polymer chain during the polymerization.

Figures 5(a) and (b) show the TEM images of PANI-DP and PANI-DF taken at 20 and $50 \mathrm{~nm}$ respectively, and the inset figures in the images of $20 \mathrm{~nm}$ show the electron diffraction of the two composites. The characteristic fibrillar structures are clearly visible in PANI-DP but are smeared 


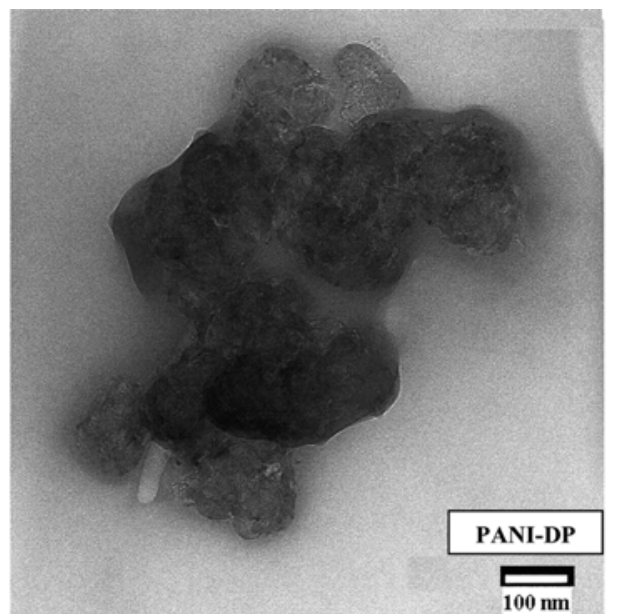

(a)

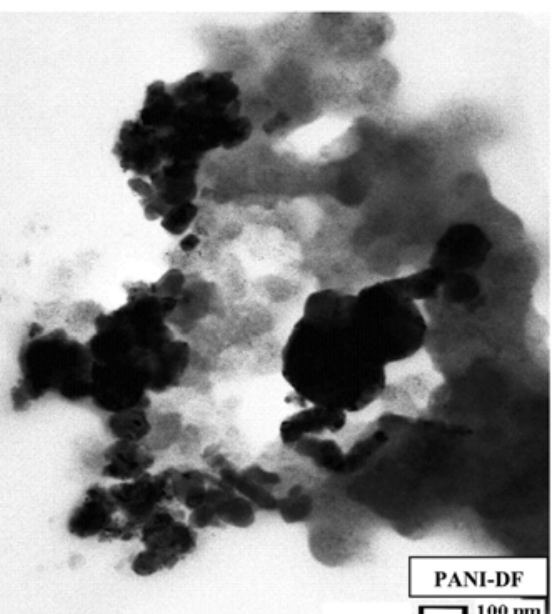

(b)

Figure 4. TEM images for (a) PANI-DP and (b) PANI-DF composites at $100 \mathrm{~nm}$.
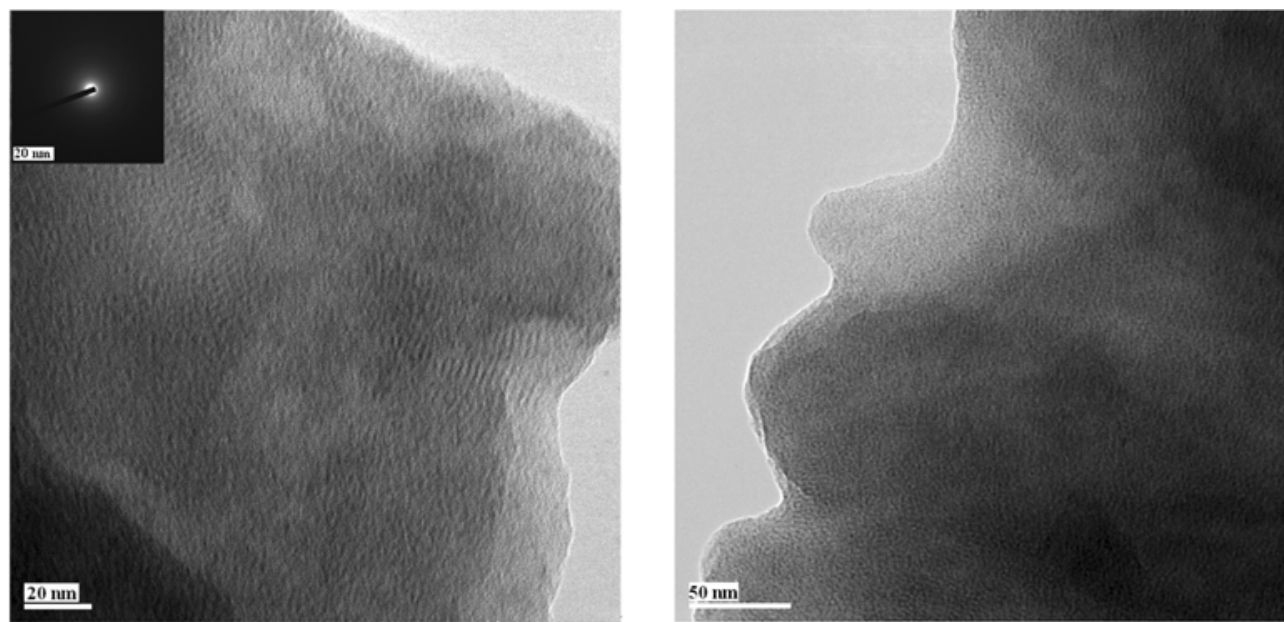

(a)
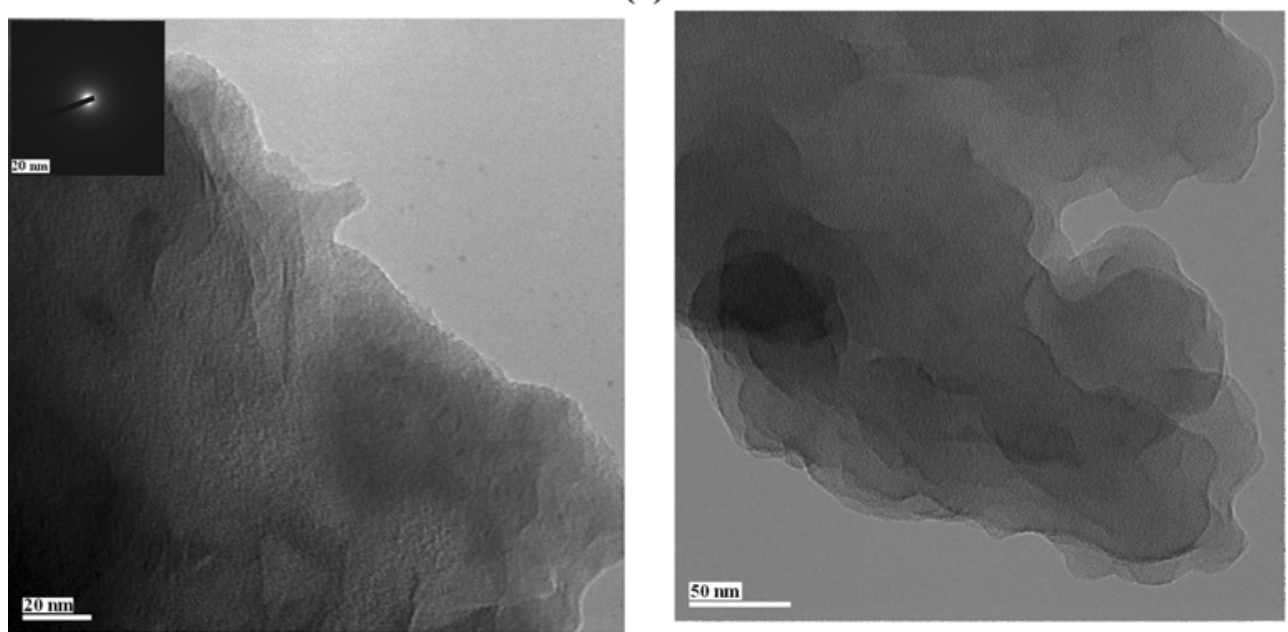

(b)

Figure 5. TEM image of PANI-DP (a) and PANI-DF (b), the insets in both figures show electron diffraction patterns of the same composites.

and darkened in PANI-DF, possibly due to the encapsulation of DBSA in PANI. The morphological changes may be attributed to the different microenvironments for the polymerization process at different oxidizing agents. The spec- 


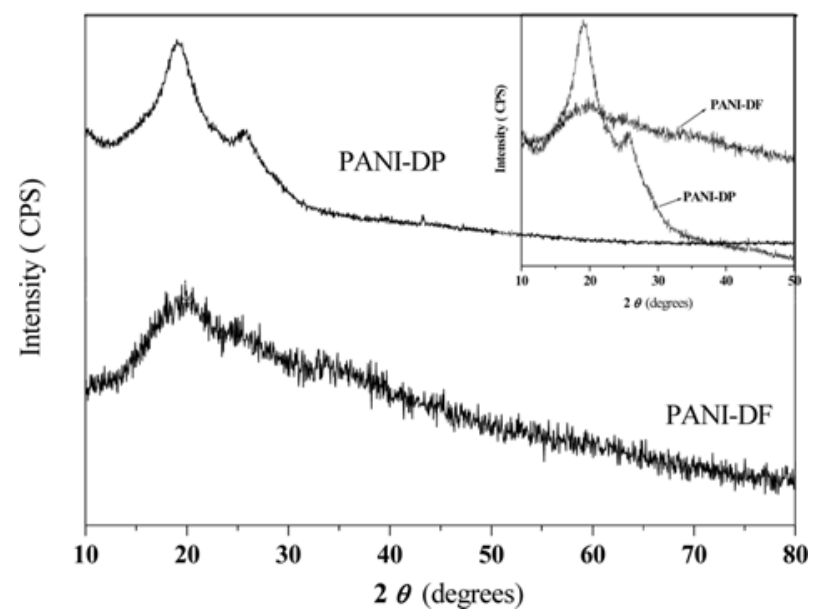

Figure 6. XRD patterns of PANI-DP and PANI-DF composites and the inset figure shows the extended portion of the same pattern.

tral characterization and the image analysis studies show that there is a morphological change in both the composites leading to the enhancement of crystallinity of PANI-DP. On the other hand, PANI-DF exhibits amorphous form with smooth and uniform surface. During the synthesis process the anilinium-DBSA complex forms micro-fabrillar network and the complex form is restored in PANI-DP. In PANI-DF the complex form of anilinium-DBSA tends to form a smooth and uniform surface.

X-ray Diffraction Studies. Figure 6 shows the XRD patterns for PANI-DP and PANI-DF and inset figure shows an extended portion of the XRD showing a difference in the diffraction patterns of the both the composites. In the diffraction pattern of PANI-DP shows two broad peaks at about 25 and 19 in the $2 \theta$ value. Whereas the PANI-DF shows a broad peak at around 25 is a characteristic diffraction pattern for PANI. The appearance of broad peaks in PANI-DP may be attributed to the residual ordered DBSA after doping. ${ }^{28,29}$ This is suggesting some differences in the composition of the samples. As evidenced by SEM images this may be due to some kind of interaction may be physical or chemical in the complex anilinium-DBSA under the condition obtained by the use of two different oxidants. This interaction at this point seems to be very difficult to analyze and a detailed study of these two composites is needed. The PANI-DP forms an organized layered like structures in which stacks of charged polyaniline backbones are spaced by the alkyl tails of the DBSA dopant. Thus, we can assume that the appearance of two peaks in PANI-DP may be due to long DBSA alkyl chains which are non-uniformly spaced in between the polymer main chains. ${ }^{30}$ We suggest that the DBSA is acting as a plasticizer that induces crystalline nature to PANI-DP composite and this property might hinder in PANI-DF.

Thermogravimetric Analysis. Figure 7 depicts the ther-

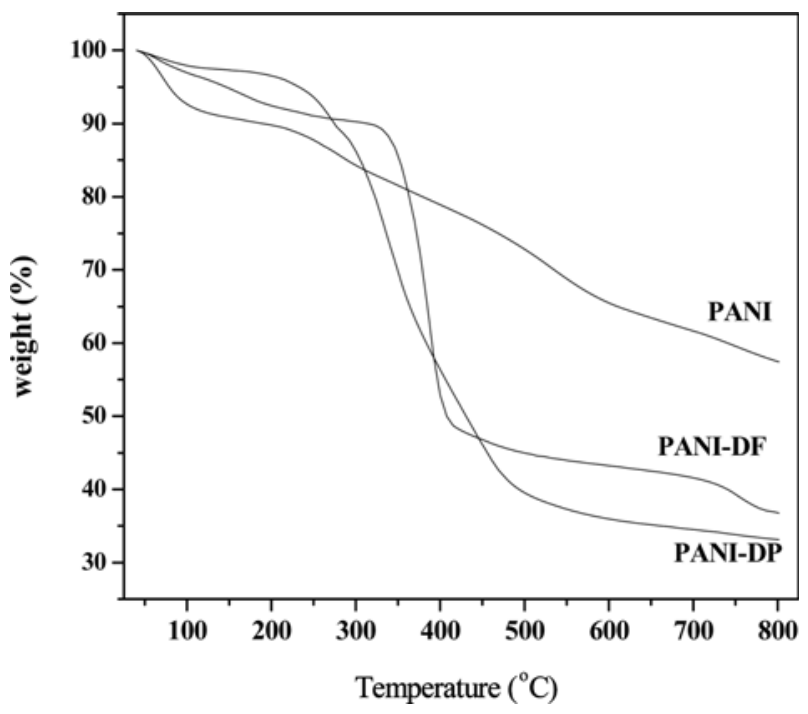

Figure 7. TGA thermograms of PANI-DP and PANI-DF composites recorded under $\mathrm{N}_{2}$ atmosphere at a heating rate of $10{ }^{\circ} \mathrm{C} / \mathrm{min}$.

Table I. Comparison of the Degradation Behavior of PANIDP and PANI-DF Composite Films

\begin{tabular}{ccccc}
\hline \multirow{2}{*}{ Composite } & \multicolumn{4}{c}{ Weight Loss (wt\%) } \\
\cline { 2 - 5 } & $300{ }^{\circ} \mathrm{C}$ & $400{ }^{\circ} \mathrm{C}$ & $500{ }^{\circ} \mathrm{C}$ & $700{ }^{\circ} \mathrm{C}$ \\
\hline PANI & 84 & 79 & 73.0 & 62.0 \\
PANI-DP & 86.17 & 56.38 & 39.5 & 34.5 \\
PANI-DF & 90.25 & 52.93 & 45.0 & 41.56 \\
\hline
\end{tabular}

mograms for PANI and for the composites of PANI-DP and PANI-DF. If we observe the curves, the PANI is more stable than the two composites. The major stages of the decomposition processes for the above three studied materials are summarized in Table I. The early stages of decomposition temperature between $100-300{ }^{\circ} \mathrm{C}$ are not clearly observed in all the three curves. There are four major stages of the decomposition process for the PANI. ${ }^{27}$ The first weight loss below $100{ }^{\circ} \mathrm{C}(\sim 7 \%)$ is a result of the release of moisture/ free water. The polymer is thermally stable up to $250^{\circ} \mathrm{C}$. After this temperature, the polymer starts to degrade slowly. The second and third stages in the temperature range 200$600{ }^{\circ} \mathrm{C}$ are associated with the decomposition of organic substances present in the PANI. Above $600{ }^{\circ} \mathrm{C}$, the polymer degrades rapidly. As for PANI-DP, a small weight loss ( 2\%) up to around $100^{\circ} \mathrm{C}$ is presumably due to the elimination of water and other volatiles. The weight loss occurring between 100 and $500{ }^{\circ} \mathrm{C}(\sim 59 \%)$ is approximately the weight fraction of DBSA; therefore it is attributed mainly to the dopant loss. It has been suggested that the lower temperature decomposition between 100 and $300{ }^{\circ} \mathrm{C}$ is induced by the excess DBSA, and the higher one between 300 and $500{ }^{\circ} \mathrm{C}$ is by the bound DBSA. Above $400{ }^{\circ} \mathrm{C}$, the polymer itself decomposes. ${ }^{31,32}$ 
From the TGA data we can also calculate how many aniline units are doped with one DBSA in PANI-DP and PANI-DF. As shown in the figure, the moisture and DBSA contents in PAN-DP are approximately $10 \%\left(\Delta Y_{1}\right)$ and $49 \%$ $\left(\Delta Y_{2}\right)$, respectively. Therefore the weight percentage of PANI should be around $51 \%$. In order to estimate how many aniline repeat units are doped with one DBSA molecule in PANI-DBSA, eq. (1) is set up: $:^{33}$

$$
\frac{100-\Delta Y_{1}-\Delta Y_{2}}{\Delta Y_{2}}=\frac{M_{1} \times X}{M_{2} \times 1}
$$

where $\Delta Y_{1}$ and $\Delta Y_{2}$ represent the weight losses due to moisture and DBSA, respectively. $M_{1}$ and $M_{2}$ represent the molecular weights of aniline and DBSA, respectively. $X$ represents the number of aniline repeat units. Therefore, using eq. (1), we substitute $\Delta Y_{1}$ and $\Delta Y_{2}, M_{1}$ and $M_{2}$ with $4.5,41,92$, and 326 , respectively. We will find that the value of $X$ is around $4.8 \pm 0.2$. It implies that there are approximately 3.0 aniline repeat units on the average, doped with one DBSA molecule in the PANI-DP composite. In a similar fashion, for PANI-DF we substitute $\Delta Y_{1}$ and $\Delta Y_{2}, M_{1}$ and $M_{2}$ with 10,49 , 92, and 326, respectively. The value of $X$ in this case is around $3.0 \pm 0.2$. It implies that there are, on the average, approximately 4.8 and 3.0 units of aniline repeat units doped with one DBSA molecule in PANI-DP and PANI-DF respectively. The high amount of doping of DBSA on the backbone of PANI results in its dark green color. The difference in the degradation temperatures of PANI-DP and PANI-DF is attributed to the dropping of dopants at higher temperatures. The TGA thermogram of the PANI-DP has more thermal stability as compared to PANI-DF. The onset of decomposition temperature of bound DBSA has increased by a factor of $\sim 20-30{ }^{\circ} \mathrm{C}$, in PANI-DP relative to the PANI-DF. As one

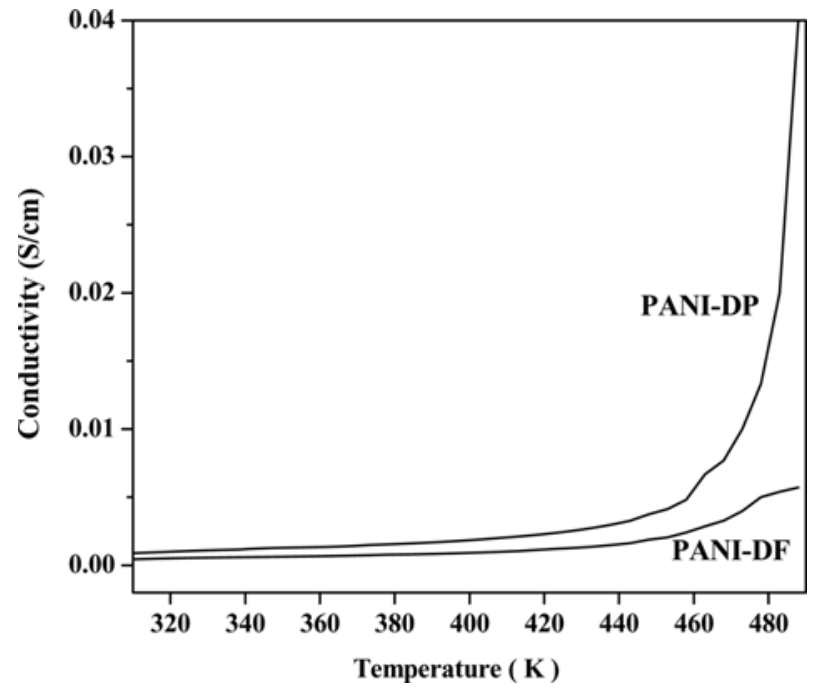

Figure 8. Temperature dependendent conductivity of PANI-DP and PANI-DF composites. can see from the SEM and TEM images, the PANI-DP possesses a fibrillar like structure where as PANI-DF has a smooth and uniform (powder) structure. This behavior may be expected because of the difference in the morphology of these two composites.

Temperature Dependent DC Conductivity. Figure 8 shows the temperature dependent dc conductivity of PANI-DP and PANI-DF composite films were measured in the range of $300-500 \mathrm{~K}$. It was found that the conductivity of both samples increases with increasing temperature indicating a semi-conducting behavior of the composite films and values conductivity lies in the range of $10^{-4}-10^{-2}$ and $10^{-4}-10^{-3} \mathrm{~S} / \mathrm{cm}$ for PANI-DP and PANI-DF, respectively. This may be due to the structure of PANI-DP which has a well-organized layered structure, and this structure is retained at relatively higher temperature. As a result the increase in conductivity is observed at relatively higher temperature ranges. This may be facilitated by the initiator APS, and may not be by FC. The change in morphology has a great influence on the conductivity of the two composite films. ${ }^{34}$

According to Zilberman ${ }^{35}$ and Leyva et al., ${ }^{36}$ the percolation threshold of the PANI-DBSA structure forms an interconnected matrix through the insulator matrix, allowing the electric conductivity of the mixture to the optimum level. The level of interaction between the PANI and DBSA matrix determines the blend morphology and, thus, its electrical conductivity. The two polymeric components are dispersed within the polymer matrix enhancing the conducting paths in the composites. PANI-DBSA presents as two different structures and these morphologies are found in this work.

\section{Conclusions}

PANI-DBSA composites were synthesized by oxidative chemical polymerization of aniline at low temperature using the initiators APS and $\mathrm{FeCl}_{3}$. The chemical interaction and surface modification were studied. The results suggest that the physical and chemical properties of the PANI can be altered by varying the oxidants in the presence of a surfactant DBSA. The change in the morphology may be due to the arrangement of polymer chain under different favorable conditions in the presence of APS or $\mathrm{FeCl}_{3}$. This is probably due to the conformational rearrangement of anilinium-DBSA complex under different oxidizing conditions as it adopts a different morphology, resulting in the change of physical and chemical properties in the presence of DBSA copolymer. The interesting combination of PANI and DBSA phase structure and morphology makes the polymer-surfactant system useful in fabricating multifunctional materials in bulk quantity for future technological applications..$^{37,38}$

Acknowledgement. The authors are grateful for the Korea Research Foundation Grant funded by the Korean Government (MOEHRD) (KRF-00042007070-00). 


\section{References}

(1) E. M. Genies, A. Boyle, M. Lapkowski, and C. Tsintavis, Synth. Met., 36, 139 (1990).

(2) C. M. Finch, S. Srichantaropass, S. W. Bailey, I. M. Grace, V. M. García-Suárez, and C. J. Lambert, J. Phys.: Condens. Matter., 20, 22203 (2008).

(3) A. Skotheim, Handbook of Conducting Polymers, New York, Marcel Dekker, 1986, Vol. 1.

(4) H. S. Nalwa, Handbook of Organic Conductive Molecules and Polymers, New York, Wiley, 1997, Vol. 4.

(5) M. G. Guenza, J. Phys.: Condens. Matter., 20, 33101 (2008).

(6) C. R. Martin, Chem. Mater, 8, 1739 (1996).

(7) I. D. Norris, M. M. Shaker, F. K. Ko, and A. G. MacDiarmid, Synth. Met., 114, 109 (2000).

(8) X. Y. Zhang, W. J. Goux, and S. K. Manohar, J. Am. Chem. Soc., 126, 4502 (2004).

(9) J. X. Huang, S. Virji, B. H. Weiller, and R. B. Kaner, J. Am. Chem. Soc., 125, 314 (2003).

(10) Z. Wei, Z. Zhang, and M. Wan, Langmuir, 18, 917 (2002).

(11) Z. Wei and M. Wan, Adv. Mater., 14, 1314 (2002).

(12) D. Wang and F. Caruso, Adv. Mater., 13, 350 (2001).

(13) W. L. Macknight, E. A. Ponomarenko, and D. A. Tirrell, Acc. Chem. Res., 31, 781 (1998).

(14) T. Jana and A. K. Nandi, Langmuir, 17, 5768 (2001).

(15) T. Jana, J. Chatterjee, and A. K. Nandi, Langmuir, 18, 5720 (2002).

(16) J. Ruokolainen, R. Makinen, M. Torkkeli, T. Makela, R. Serimaa, G. ten Brinke, and O. Ikkala, Science, 280, 557 (1998).

(17) D. Tsotcheva, T. Tsanov, L. Terlmezyam, and S.Vassilev, J. Therm. Anal., 63, 133 (2001).

(18) W. Jia, E. Segal, D. Kornemandel, Y. Lamhot, M. Narkis, and A. Siegmann, Synth. Met., 128, 115 (2002).

(19) C. Basavaraja, Y. Veeranagouda, K. Lee, R. Pierson, S. H. Park, E. J. Jeon, and D. S. Huh, Eur. Polym. J. (submitted for publi- cation) (2008).

(20) C. Basavaraja, R. Pierson, and D. S. Huh, J. Appl. Polym. Sci., 108, 1070 (2008).

(21) C. Basavaraja, R. Pierson, T. K. Vishnuvardhan, and D. S. Huh, Eur. Polym. J., 44, 1556 (2008).

(22) M. Antonietti, D. Radloff, U. Niesner, and H. W. Spiess, Macromol. Chem. Phys., 197, 2713 (1996).

(23) M. Antoniett, A. Wenzel, and A. Thunemann, Langmuir, 12, 2111 (1996).

(24) P. Chowdhury and B. Saha, J. Appl. Polym. Sci., 103, 1626 (2007).

(25) Y. Z. Wang, Y. C. Hsu, L. C. Chou, and K. H. Hsieh, J. Polym. Res., 11, 127 (2004).

(26) A. Gok, B. Sari, and M. Talu, Synth. Met., 142, 41 (2004).

(27) A. Gok, M. Omastov, and A. Y. Gul, Synth. Met., 157, 23 (2007).

(28) A. A. Athawale, M. V. Kulkarni, and V. V. Chabukswar, Mater. Chem. Phys., 73, 106 (2002).

(29) S. A. Chen and H. T. Lee, Synth. Met., 47, 233 (1992).

(30) M. Wan, M. Li, J. Li, and Z. Liu, J. Appl. Polym. Sci., 53, 131 (1994).

(31) D. Das, S. Kar, S. Chakraborty, D. Chakraborty, and S. Gangopadhyay, J. Appl. Polym. Sci., 69, 841 (1998).

(32) P. R. Somani, R. Marimuthu, and A. B. Mandale, Polymer, 42, 2991 (2001).

(33) C. H. Chen, J. Polym. Res., 9, 195 (2002).

(34) M. Wan, M. Li, J. Li, and Z. Liu, J. Appl. Polym. Sci., 53, 131 (1994).

(35) M. Zilberman, G. I. Titelman, A. Siegmann, Y. Haba, M. Narkis, and D. Alperstein, J. Appl. Polym. Sci., 66, 243 (1998).

(36) M. E. Leyva, G. M. O. Barra, and B. G. Soares, Polímeros, 12, 197 (2002).

(37) J. K. Choi, Y. W. Kim, and J. H. Koh, Marcromol. Res., 15, 553 (2007).

(38) H. Namgoong, D. J. Woo, and S. H. Lee, Marcromol. Res., 15, 633 (2007). 